28

\title{
Bayesian Mixture Models for Gene Expression and Protein Profiles
}

\author{
Michele Guindani, Kim-Anh Do, Peter Müller and Jeff Morris \\ M.D. Anderson Cancer Center
}

\section{Introduction}

We review the use of semi-parametric mixture models for Bayesian inference in high throughput genomic data. We discuss three specific approaches for microarray data, for protein mass spectrometry experiments, and for SAGE data. For the microarray data and the protein mass spectrometry we assume group comparison experiments, i.e., experiments that seek to identify genes and proteins that are differentially expressed across two biologic conditions of interest. For the SAGE data example we consider inference for a single biologic sample.

Several aspects of data analysis for microarray and other high throughput gene and protein expression experiments give rise to mixture models. One important application of mixture models is for flexible modeling of sampling distributions. This is attractive, for example, when the number of genes on a microarray is the relevant sample size, thus allowing flexible semi-parametric representations. Such approaches are discussed, among others, in Broet P (2002), Dahl (2003), or Tadesse et al. (2005). The latter exploit the clustering implicitely defined by the mixture model. Also, see Dahl (2006); Tadesse et al. (2006) in this volume. Related non-Bayesian approaches using mixture models have been discussed, for example, in Pan et al. (2002) and McLachlan, Bean, and Peel (2002) KIM: reference?. In this chapter we review three approaches that are typical of this literature. In Section 2 we discuss the use of Dirichlet process mixtures for model based inference about differential gene expression. In Sectin 3 we describe a mixture of Beta model for the mass/charge spectrum in MALDI-TOF mass spectrometry experiments. In Section 4 we introduce a semiparametric mixture of Poisson model for SAGE data.

Another important class of applications for mixture models in data analysis for high throughput gene expression data are finite mixtures, with each term in the mixture corresponding to a different condition of interest. A typical example is the model used in Parmigiani et al. (2002) who construct a sampling model for observed gene expression in microarray experiments as a mixture of three terms corresponding to normal, under- and over-expression. Newton et al. 


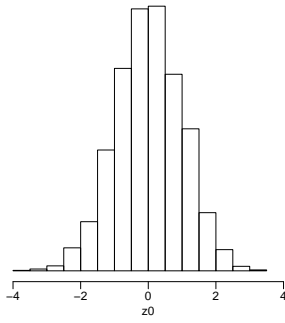

$p_{0} \cdot f_{0}$

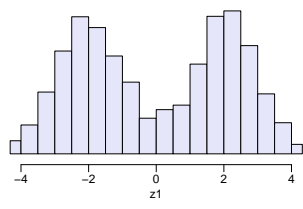

$\left(1-p_{0}\right) \cdot f_{1}$

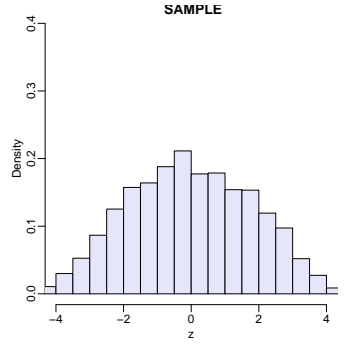

$f$

Figure 1: Hypothetical distribution of difference scores for non-differentially expressed (left, $f_{0}$ ) and differentially expessed genes (center, $f_{1}$ ), and the observed mixture (right, $f$ ).

(2001) define a Gamma/Gamma hierarchical model with a mixture induced by an indicator for ties between two biologic conditions of interest.

In this chapter we only focus on the use of semi-parametric mixtures to represent an unknown sampling model, and will not discuss the other type of mixture models.

\section{A Non-parametric Bayesian model for Differ- ential Gene Expression}

We consider inference for microarray group comparsion experiments. Assume that the data has been summarized as a set of difference scores, $z_{i}, i=1, \ldots, n$, for $n$ genes. The difference score $z_{i}$ could be, for example, a two-sample tstatistic for observed fluorescence intensities for gene $i$ in samples under two biologic conditions of interest. See Efron et al. (2001) for a discussion of appropriate data pr-eproscesing. We assume that the set $i=1, \ldots, n$ of genes is partitioned into a subset of differentially expressed genes and non-differentially expressed genes. Inference proceeds by assuming that for differentially expressed genes, the difference scores $z_{i}$ arise by independent sampling from some unknown distribution $f_{1}$; for non-differentially expressed genes, $z_{i}$ are independent samples from an unknown distribution $f_{0}$. For a reasonable choice of difference scores, the distribution $f_{0}$ should be a unimodal distribution centered at zero. The distribution $f_{1}$ should be a bimodal distribution with symmetric modes to the left and right of zero corresponding to over- and underexpressed genes. Figure 1 show possible histograms for observed difference scores generated from $f_{0}$ and $f_{1}$. Of course, the partition into differentially and non-differentially expressed genes is unknown. Thus, instead of samples from $f_{0}$ and $f_{1}$, we can only work with the sample $z_{i}, i=1, \ldots, n$, generated from a mixture of $f_{0}$ and $f_{1}$. Let $p_{0}$ denote the unknown proporition of non-differentially genes. We assume

$$
z_{i} \stackrel{i i d}{\sim} f(z)=p_{0} f_{0}(z)+\left(1-p_{0}\right) f_{1}(z), \quad i=1, \ldots, n .
$$


The main goal of inference in the two group comparison microarray experiment can be formally described as the deconvolution of (1). We introduce a latent indicator variables $r_{i} \in\{0,1\}$ to rewrite (1) equivalently as a hierarchical model

$$
\begin{aligned}
p\left(z_{i} \mid r_{i}=j\right) & =f_{j}\left(z_{i}\right) \\
\operatorname{Pr}\left(r_{i}=0\right) & =p_{0}
\end{aligned}
$$

The latent variable $r_{i}$ can be interpreted as indicator for gene $i$ being differentially expressed. Efron et al. (2001) propose cleverly chosen point estimates for $p_{0}, f_{0}$ and $f_{1}$ and report the implied inference for $r_{i}$. To develop the point estimate they introduce an additional set of difference scores, $z_{i}, i=n+1, \ldots, 2 n$. The additional difference scores are generated using the same original data, but deliberately computing difference scores for samples under the same biologic conditions. Thus,

$$
z_{i} \sim f_{0}\left(z_{i}\right), \quad i=n+1, \ldots, 2 n
$$

for this additional null sample.

In Do et al. (2005) we propose a model-based semiparametric Bayesian approach to inference in this problem. We recognize $f_{0}, f_{1}$ and $p_{0}$ as unknown quantities and proceed by defining a suitable prior probability model. Probability models for unknown functions, including distributions such as $f_{0}$ and $f_{1}$ in this problem, are known as non-parametric Bayesian models. See, for example Müller and Quintana (2004) for a recent review of non-parametric Bayesian inference. The term "non-parametric" is a misnomer, as the random functions are infinite dimensional parameters. However, the name is traditionally used because implied posterior inference closely resembles inference under classical non-parametric methods.

In chosing a prior probability model for $f_{0}$ and $f_{1}$ we face two competing aims. On one hand we wish to generalize traditional parametric models, like a normal sampling model. On the other hand we want to retain as much computational simplicity as possible. This leads us to use a mixture of normal model, with a non-parametric prior on the mixing measure. Inference under this model is almost as straightforward as under a simple normal model, yet, subject to some technical constraints, the mixture of normal model can approximate arbitrary sampling distributions. As probabilty model for the mixing measure we use a Dirichlet process (DP) prior (Ferguson, 1973; Antoniak, 1974). For reasons of computational simplicity and ease of interpretation the DP prior is one of the most widely used non-parametric Bayes models. The DP model has two parameters, a base measure and a total mass parameter. We write $G \sim D P\left(G^{\star}, M\right)$ to indicate that $G$ has a DP prior with a base measure $G^{\star}$ and total mass $M$. The base measure has the interpretation as mean measure, in fact $E(G)=G^{\star}$. The total mass parameter can be interpreted as a precision parameter. The larger $M$, the closer the random $G$ will be to $G^{\star}$.

In sumary, we assume the following model. Let $N(z ; m, s)$ denote a normal distribution for the random variable $z$, with moments $(m, s)$. We define a 
probability model for the random distributions $f_{0}$ and $f_{1}$ as:

$$
\begin{gathered}
f_{j}(z)=\int N(z ; \mu, \sigma) d G_{j}(\mu) \\
G_{j} \sim D P\left(G_{j}^{\star}, M\right) .
\end{gathered}
$$

One of the critical properties of the DP prior is that a DP generated random measure is almost surely discrete. Thus the integral in (3) is simply a sum over all point masses in $G_{j}$. Mixture models with respect to a DP, such as (3), are known as mixture of DP (MDP) models and are widely used in non-parametric Bayesian inference. See, for example, MacEachern and Müller (2000) for a review of such models.

We complete the model given by the likelihood (2) and prior (3) with a hyperprior on the base measures $G_{j}^{\star}$. We assume $G_{0}^{\star}=N\left(0, \tau^{2}\right)$ with a conjugate inverse Gamma hyperprior on $\tau^{2}$, and $G_{1}^{\star}=\frac{1}{2} N\left(-b, \tau^{2}\right)+\frac{1}{2} N\left(b, \tau^{2}\right)$ with a conjugate normal hyperprior on $b$. Finally, we assume a Beta prior for $p_{0}$, $p_{0} \sim B e(\alpha, \beta)$. The hyperparameters $\alpha, \beta, M$ are fixed.

Inference in the proposed model is implemented by Markov chain Monte Carlo (MCMC) simulation. See Do et al. (2005) for a detailed description of the posterior MCMC algorithm. A direct implication of the model (2) and (3) is that the marginal posterior probability of differential expression, $\operatorname{Pr}\left(r_{i}=1 \mid\right.$ data $)$, is the same for all genes with equal difference score $z_{i}$. Thus posterior inference can be summarized as a function $\operatorname{Pr}\left(r_{i}=1 \mid z_{i}=z\right.$, data $)$. Starting with model (2), a straightforward use of Bayes theorem shows

$$
\operatorname{Pr}\left(r_{i}=0 \mid z_{i}=z, f_{0}, f_{1}, p_{0}\right)=p_{0} f_{0}(z) /[\underbrace{p_{0} f_{0}(z)+\left(1-p_{0}\right) f_{1}(z)}_{f(z)}] .
$$

Let $P_{1}=p_{0} f_{0} / f$. Then the posterior expectation $\bar{P}_{1}=E\left(P_{1} \mid\right.$ data $)$ is exactly the desired marginal posterior probability of differential expression, $\bar{P}_{1}=\operatorname{Pr}\left(r_{i}=1 \mid z_{i}=z\right.$, data $)$. Figure 2 shows posterior inference for a simulation experiment. The figure shows the simulation truth, the reported posterior mean curve $\bar{P}_{1}(z)$, and pointwise posterior credible intervals for $P_{1}(z)$.

\section{A Mixture of Beta Model for MALDI-TOF Data}

Matrix assisted laser disorption - time of flight (MALDI-TOF) experiments allow to simultaneously measure abundance for a large number of proteins. Details of the experimental setup are described, for example, in Baggerly et al. (2003). See also Baggerly et al. (2006), in this volume. Briefly, the biological sample for which we wish to determine protein abundance is fixed in a matrix. A laser beam is used to break free and ionize individual protein molecules. The experiment is arranged such that ionized proteins are exposed to an electric field that accelerates molecules along a flight tube. On the other end of the flight 

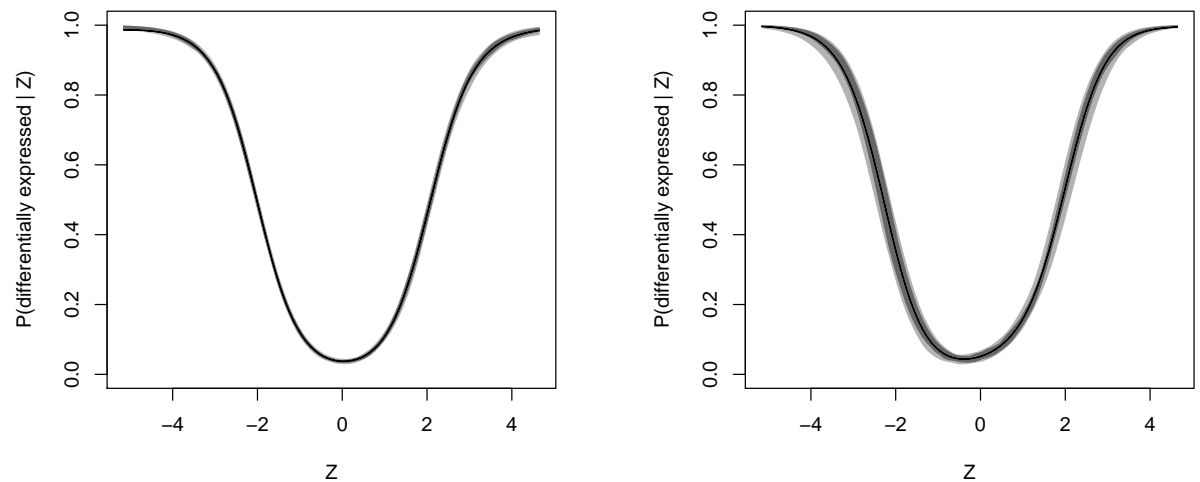

Figure 2: $\bar{P}_{1}(z)$ : posterior mean probability of differential expression as a function of the observed difference score $z$ (solid black line). The left figure conditions on the full data, $z_{i}, i=1, \ldots, 2 n$, including the null data. The right figure does not make use of the null data, conditioning only on $z_{i}, i=1, \ldots, n$. The dark grey shaded band shows the central $50 \%$ posterior density interval. Light grey shows a $75 \%$ posterior interval The dark and light grey shaded areas are very narrow and can hardly be distinguished from the posterior mean curve.

tube, molecules hit a detector that records a histogram of number of molecules that hit over time. Assumig that all ionized molecules carry a unit charge, the time of flight is deterministically related to the molecule mass. The histogram of detector events over time can therefore be be changed to a histogram of detector events over protein masses. Allowing for multiple charges, the mass scale is replaced by a scale of mass/charge ratios. The histogram of detector events is known as mass/charge spectrum. Figure 3 shows typical spectra.

plot of one spec plot of other spec

Figure 3: Spectra for a normal samples (left) and a tumor samples (right), on grid of size $I=60,000$. (plots are big; i have removed them temp'ly -simply two spectra)

Ideally, each protein that is present in the original probe should correspond to a peak in the spectrum. Because of the random initial velocities when proteins are ionized by the laser impact we would expect to see peaks rather than sharp lines even in an idealized experiment. Many additional artifacts of the experiment add to the idealized description, leading to an additional baseline that adds to the protein peaks. See the data shown in 3.

Assume we observe spectra for experiments $k=1, \ldots, K$. Let $y_{k}\left(m_{i}\right)$ denote 
the recorded count for sample $k$ at mass/charge grid point $m_{i}$, and let $f_{k}\left(m_{i}\right)$ denote the assumed underlying cleaned spectra corresponding to detected proteins only. The desired inference about the unknown protein abundance in the original probes can be formalized as (i) removing noise and baseline from the observed spectra $y_{k i}$ to impute $p_{k}$; (ii) finding peaks in $p_{k}$; and (iii) reporting the relative sizes of these peaks. The relative size of the peaks corresponds to the relative abundance of the corresponding protein in the probe. If samples are collected under different biologic conditions we need additional inference about different versus equal abundance of different proteins.

In Müller et al. (2005) we develop a non-parametric Bayes model to allow such inference. Based on the above stylized description of the experiment we consider $y_{k}$ as the empirical histogram of detector events. We represent it as a mixture of a baseline $B_{k}$ corresponding to detector noise, protein fragments, etc., and a cleaned spectrum $f_{k}$.

$$
p_{k}(m)=p_{0 k} B_{k}(m)+\left(1-p_{0 k}\right) f_{k}(m) .
$$

The spectrum $f_{k}$ is a sum of peaks, with each detected protein contributing a peak centered at its mass/charge value. The experimental arrangement implies a finite support for $f_{k}$. Motivated by nonparametric models for random distributions on a finite support developed in Petrone (1999) and Robert and Rousseau (2002) we use a mixture of Beta distributions to define the random distribution $f_{k}$. The location for each Beta kernel is interpreted as the mass/charge ratio of the protein giving rise to this peak. To facilitate later interpretation, we use a non-standard parametriztion of the Beta distribution. We write $B e(x ; \epsilon, \alpha)$ for a Beta kernel for the random variable $x$, with mean and standard deviation $\epsilon$ and $\alpha$ (with appropriate constraints on $\alpha$ ).

Let $x$ denote the biologic condition of sample $k$. We assume a two group comparison, i.e., $x \in\{0,1\}$. Then

$$
f_{k}(m)=\sum_{j=1}^{J} w_{x j} \operatorname{Beta}\left(m ; \epsilon_{j}, \alpha_{j}\right) .
$$

In words, the $k$-th spectrum is a mixture of Beta kernels, corresponding to $J$ distinct proteins with mass/charge values $\epsilon_{j}$. The relative weight $w_{x j}$, i.e., relative abundance of protein $j$, is assumed the same for all samples under the same biologic condition. For reasons of technical convenience we chose an identical mixture of Beta prior for the baseline $B_{k}$. Different hyperparameters reflect the fact that the baseline is much smoother than $f_{k}$ and we expect fewer terms in the mixture. $B_{k}(m)=\sum_{j=1}^{J_{k}} v_{k j} \operatorname{Beta}\left(m ; \eta_{k j}, \beta_{k j}\right)$. The sizes of the mixtures are random. We use truncated Poisson priors for $J$ and $J_{k}, k=$ $1, \ldots, K$. Baseline $B_{k}$ and mean spectrum $f_{k}$ are combined to define the random distribution of mass/charge ratios $p_{k}=p_{0 k} B_{k}+\left(1-p_{0 k}\right) f_{k}$. Following the idealized description of the experimental setup, the sampling model is random sampling from $p_{k}$. Let $y_{k}=\left(y_{k i}, i=1, \ldots, I\right)$ denote the empirical spectrum for the $k$-th sample over the grid of mass/charge values. Typically $I$ is a large, 
say 60,000 , defining a very fine grid. Let $\theta=\left(J, J_{k}, w_{x j}, v_{k j}, \epsilon_{j}, \alpha_{j}, \eta_{i}, \beta_{i}, x=\right.$ $\left.0,1, j=1, \ldots, J, k=1, \ldots, K, i=1, \ldots, J_{k}\right)$ denote the parameter vector. The likelihood is

$$
\log p\left(y_{k} \mid \theta\right)=\sum_{i=1}^{I} y_{k i} \log p_{k}\left(m_{i}\right) .
$$

Instead of the random sampling model (5) many authors use a regression likelihood, assuming normal residuals, $y_{k i} \sim N\left(p_{k}\left(m_{i}\right), \sigma^{2}\right)$. Little changes in the following discussion if we were to replace (5) by this regression likelihood.

The model is completed with a prior for the Beta parameters and the weights. For the weights $w_{x j}$ we use a hierarchical prior with indicators $\lambda_{j}$ for ties

$$
\lambda_{j}=I\left(w_{0 j}=w_{1 j}\right)
$$

Posterior inference on the $\lambda_{j}$ and the locations $\epsilon_{j}$ summarizes the desired inference on proteins that are differentially expressed across the two groups of samples.

Implementation of posterior inference requires MCMC over a varying dimension parameter space, as the dimension of the parameter space depends on the sizes $J$ and $J_{k}$ of the mixtures. We use reversible jump MCMC (RJMCMC) as proposed in Green (1995) and, specifically for mixture models, in Richardson and Green (1997). See Müller et al. (2005) for a detailed description of the MCMC algorithm.

A minor complication arises in reporting and summarizing posterior inference about distinct proteins and their mass/charge ratios. The mixture $f_{k}$ only includes exchangeable indices $j$, leading to the complication that the Beta kernel corresponding to a given protein might have different indices at different iterations of the posterior MCMC simulation. In other words, the protein identity is not part of the probability model. To report posterior inference on the mean abundance of a given protein requires additional post-processing to match Beta kernels that correspond to the same protein across iterations. We use a reasonable ad-hoc rule. Any two peaks $j$ and $h$ with a difference in masses below a certain threshold are counted as arising from the same protein. Specifically, we use the condition $\left|\epsilon_{j}-\epsilon_{h}\right|<0.5 \alpha_{j}$ to match peaks. Here $j$ indexes the peak that was imputed in an earlier MCMC iteration than the peak $h$. The problem of reporting inference related to the terms in a mixture is known as the label switching problem (C. C. Holmes and Stephens, 2005).

Figure 4 summarizes estimated masses and abundance of detected proteins.

\section{A Semi-Parametric Mixture Model for SAGE Data}

Consider data from a SAGE (Serial Analysis of Gene Expression) experiment. See Baggerly et al. (2006) for a description of the experimental setup, and the 


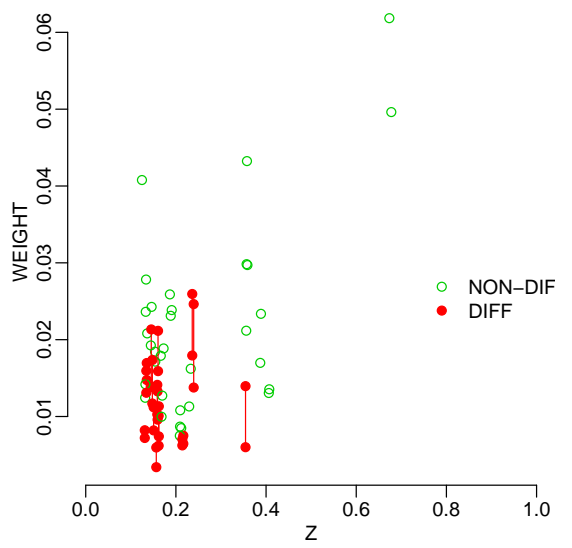

Figure 4: Posterior mean abundance of detected proteins. All peaks with posterior probability of differential expression greater $50 \%$ are marked as solid dots, with a line combining $\bar{w}_{0 j}=E\left(w_{0 j} \mid\right.$ data $)$ and $\bar{w}_{1 j}$.

nature of the data. We consider inference for data from one biologic sample. Let $y_{i}, i=1, \ldots, k$, denote observed tag frequencies for $k$ distinct transcripts. Let $n=\sum y_{i}$ denote the total number of recorded transcripts, and let $\pi_{i}$ denote the unknown true abundance of the $i$-th transcript in the probe. For large $y_{i}$, the empirical frequency $\widehat{\pi}_{i}=y_{i} / n$ is an appropriate point estimate for $\pi_{i}$. The associated uncertainty, formalized as variance of the maximum likelihood estimator or as posterior standard deviation in a suitable model, is negligible. However, for scarce tags, the empirical frequency includes considerable sampling variability, and more elaborate estimates are desireable. Also, when the data includes samples across different biologic conditions, the inference goal might not be restricted to estimating the transcript frequencies. For discrimination and classification additional inference about differences in transcript frequencies, and related probability statements are required. In addition to inference on $\pi_{i}$ for a specific tag $i$, one might be interested in the distribution of tag frequencies across different transcripts. This can be achieved by model-based posterior inference.

Morris et al. (2003) introduce an approach that is based on a hierarchical model with a mixture of two Dirichlet distributions as population distribution prior for the $\pi_{i}$. See Morris et al. (2006) in this volume for a review of this approach. Building on this model, we introduce a semi-parametric Bayesian mixture model, replacing the two-component mixture of Dirichlet distributions by an unknown random measure, with a nonparametric Bayesian prior model.

For the following model construction it is convenient not to condition on $n$. In other words, instead of assuming that the set of observed counts arise as a multinomial sample with cell frequencies $\pi_{i}$, we assume that, conditional on 
hyperparameters, the counts $y_{i}$ arise as indpendent samples from some appropriate probability model. Specifically, we assume that the counts $y_{i}$ are sampled from a mixture of Poisson model. Let $\operatorname{Poi}(x ; \lambda)$ denote a Poisson distribution for the random variable $x$ with parameter $\lambda$. We assume

$$
y_{i} \sim \int \operatorname{Poi}\left(y_{i} ; \lambda\right) \mathrm{d} G(\lambda),
$$

$i=1, \ldots, n$, independently conditional on $G$. We specify a prior distribution for the mixture model by assuming a nonparametric prior on the mixing measure, chosing a DP prior as in (3),

$$
G \sim D P\left(G^{\star}, M\right) .
$$

The mixture model can alternatively be written as a hierarchical model

$$
y_{i} \mid \lambda_{i} \sim \operatorname{Poi}\left(\lambda_{i}\right) \text { with } \lambda_{i} \sim G .
$$

The discrete nature of the DP random measure $G$ implies a positive probability for ties among the $\lambda_{i}$. We denote with $L$ the number of distinct values.

A minor complication arises from the fact that $y_{i}=0$ is not observed; it is censored. Let $k_{0}$ denote the number of tags with non-zero count, i.e., the number of tags recorded in a SAGE library as shown in Baggerly et al. (2006). One could augment the model to include inference on $k, k \geq k_{0}$. Alternatively, we follow Stollberg et al. (2000), and fix $k$ by imputing a point estimate for the uknown number of unobserved tags, i.e., tags with $y_{i}=0$.

Model (6) and (7) defines a DP mixture of Poisson distributions. Such models are popular choices for non-parametric Bayesian data analysis. See, for example, MacEachern and Müller (2000) for a review of such models, including implementation of posterior inference by MCMC simulation. Choosing the base measure $G^{\star}$ to be conjugate with the Poisson distribution we define a conjugate DP mixture, greatly facilitating the MCMC implementation. Let $\operatorname{Ga}(x ; \alpha, \beta)$ denote a Gamma distribution with mean $\alpha / \beta$. We use

$$
G^{\star}(\lambda)=G a(x ; \alpha, \beta),
$$

with fixed hyperparameters $\alpha$ and $\beta$.

To illustrate the model we implemented posterior inference for a SAGE library reported in Zhang et al. (1997). The same data was used in Morris et al. (2003), and is available at http://www.sagenet.org/SAGEData/NC1.htm. It records counts for $k_{0}=17703$ distinct transcripts, with a total number of $n=\sum y_{i}=49610$ recorded tags. We use the estimte from Stollberg et al. (2000), and set $k=25336$, with $y_{i}=0$ for $i=k_{0}+1, \ldots, k$, i.e., we estimate the number of tags with censored counts $y_{i}=0$, as $\sum I\left(y_{i}=0\right)=8072$. Figure 5 a shows a histogram of observed counts $y_{i}$ in the data. Figure 6 summarizes posterior inference for the transcript abundances. The figure plots posterior mean estimates $E\left(\lambda_{i} \mid\right.$ data $)$ versus observed counts $y_{i}$. The nature of the shrinkage follows the patter reported in Morris et al. (2003). For censored 


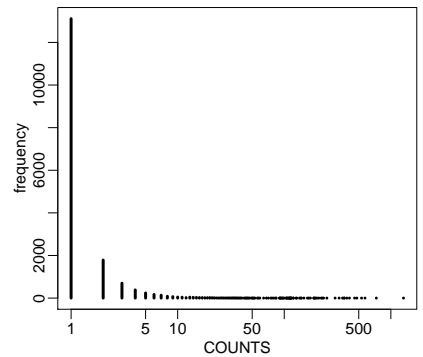

Figure 5: Observed tag counts $y_{i}$. The highly skewed nature is typial for SAGE data. Will still redo the figure in log-log scale.

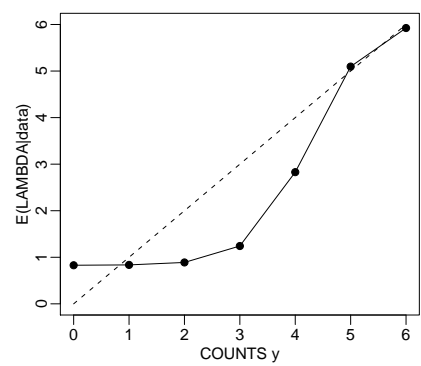

(a) $\bar{\lambda}_{i}$ vs. $y_{i}$

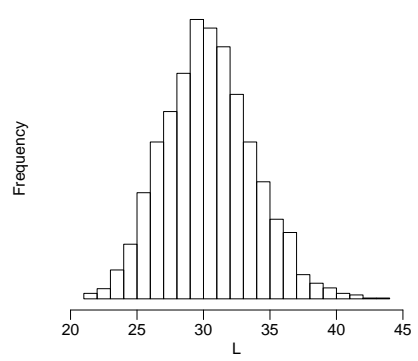

(b) $p(L \mid d t a)$.

Figure 6: Posterior means $\bar{\lambda}_{i}=E\left(\lambda_{i} \mid d t a\right)$ versus observed counts $y_{i}$ (panel a). Note the strong shrinkage for small counts. For large counts, $y_{i}>6$, posterior shrinkage quickly becomes negligible. Posterior distribution for the number of clusters $L$ (panel b). 


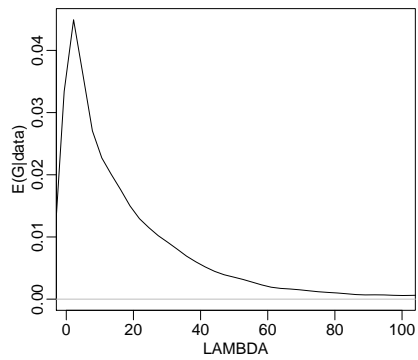

(a) $E(G \mid d t a), \lambda<100$

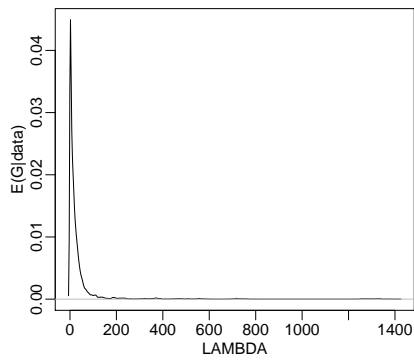

(b) $E(G \mid d t a)$

Figure 7: Estimated mixing measure $G$. The left panel zooms in on the lower end, $\lambda<100$. The highly skewed nature of $G^{\star}=E(G \mid d t a)$ reflects the same feature in the recorded data $y_{i}$.

tags, with $y_{i}=0$, the posterior mean estimate inflates the m.l.e. and reports $E\left(\lambda_{i} \mid\right.$ data $) \approx 0.9$. For rare tags with non-zero counts, posterior inference shrinks the maximum likelihood estimate. For abundant tags, posterior inference is driven only by the observed count, and $E\left(\lambda_{i} \mid d a t a\right) \approx y_{i}$. Figure 7 shows the estimated distribution of tag abundances $\lambda_{i}$.

\section{Summary}

We have illustrated the use of mixture models for Bayesian inference for gene expression and proteomics. We focused on the use of mixtures as a flexible class of distributions to parametrize random distributions. Another important use of mixtures arises in models where the submodels in the mixture correspond to different biologic conditions. Such models are extensively reviewed in other chapters in this volume.

We introduced DP mixtures of normal models to model microarray gene expression data, DP mixtures of Poissons to model tag counts in SAGE data, and location/scale mixtures of Beta kernels to represent mass/charge spectra in protein mass spectrometry experiments. The underlying theme in all three applications is the use of model-based inference, with a probability model on the random distribution (or mass/charge spectrum). This is in contrast to traditional, and very resonable, multi-step methods. If the goal is only a single point estimate of the gene expression in the microarray experiment, peak location and weight in the mass/charge spectrum, and abundance of a transcript in the SAGE data, then the model based methods are perhaps overly complex. The result will differ little from any reasonable ad-hoc method and might not justify the computational effort. The power of these methods lies in the full 
probabilistic description of all related uncertainties. But many important inference problems go beyond point estimates. For example, consider the decision problem of flagging genes for differential expression, or the problem of identifying a set of proteins that can serve as biomarker panel, or sample size choice for

a microarray experiment. A decision theoretic answer to these question relies on a description of all uncertainties in one coherent probability model.

\section{Acknowledgments}

Jeff Morris was supported by NCI grant CA-107304. Michele Guindani and Peter Müller were supported by NCI grant CA75981.

\section{References}

Antoniak, C. E. (1974), "Mixtures of Dirichlet processes with applications to Bayesian nonparametric problems," The Annals of Statistics, 2, 1152-1174.

Baggerly, K. A., Coombes, K. R., and Morris, J. S. (2006), "Bayesian Inference for Gene Expression and Proteomics," in Do et al. (2006), chap. An Introduction to High-Throughput Bioinformatics Data, pp. 1-91.

Baggerly, K. A., Morris, J. S., Wang, J., Gold, D., Xiao, L. C., and Coombes, K. R. (2003), "A comprehensive approach to analysis of MALDI-TOF proteomics spectra from serum samples," Proteomics, ?, ???-???

Broet P, Richardson S, R. F. (2002), "Bayesian hierarchical model for identifying changes in gene expression from microarray experiments," J Comput Biol., 9, $671-83$.

C. C. Holmes, A. J. and Stephens, D. A. (2005), "Markov Chain Monte Carlo Methods and the Label Switching Problem in Bayesian Mixture Modeling," Statistical Science, 20, 50-67.

Dahl, D. (2003), "Modeling differential gene expression using a Dirichlet Process mixture model," in 2003 Proceedings of the American Statistical Association, Bayesian Statistical Sciences Section, Alexandria, VA: American Statistical Association.

- (2006), "Bayesian Inference for Gene Expression and Proteomics," in Do et al. (2006), chap. Model-Based Clustering for Expression Data via a Dirichlet Process Mixture MOdel, pp. 1-91.

Do, K., Müller, P., and Tang, F. (2005), "A Bayesian mixture model for differential gene expression," Journal of the Royal Statistical Society C, 54, 627-644.

Do, K.-A., Müller, P., and Vannucci, M. (eds.) (2006), Bayesian Inference for Gene Expression and Proteomics, Cambridge University Press. 
Efron, B., Tibshirani, R., Storey, J. D., and Tusher, V. (2001), "Empirical Bayes analysis of a microarray experiment," Journal of the American Statistical Association, 96, 1151-1160.

Ferguson, T. S. (1973), "A Bayesian analysis of some nonparametric problems," The Annals of Statistics, 1, 209-230.

Green, P. J. (1995), "Reversible jump Markov chain Monte Carlo computation and Bayesian model determination," Biometrika, 82, 711-732.

MacEachern, S. N. and Müller, P. (2000), "Efficient MCMC Schemes for Robust Model Extensions using Encompassing Dirichlet Process Mixture Models," in Robust Bayesian Analysis, eds. Ruggeri, F. and Ríos-Insua, D., New York:Springer-Verlag, pp. 295-316.

Morris, J. S., Baggerly, K. A., and Coombes, K. R. (2003), "Bayesian Shrinkage Estimation of the Relative Abundance of MRNA Transcripts Using SAGE," Biometrics, 59, 476-486.

- (2006), "Bayesian Inference for Gene Expression and Proteomics," in Do et al. (2006), chap. Shrinkage Estimation for SAGE Data using a Mixture Dirichlet Prior, pp. 1-37.

Müller, P. and Quintana, F. A. (2004), "Nonparametric Bayesian Data Analysis," Statistical Science, 19, 95-110.

Newton, M. A., Kendziorsky, C. M., Richmond, C. S., R., B. F., and Tsui, K. W. (2001), "On differential variability of expression ratios: improving statistical inference about gene expression changes from microarray data," Journal Computational Biology, 8, 37-52.

Pan, W., Lin, J., and Le, C. T. (2002), "How many replicates of arrays are required to detect gene expression changes in microarray experiments? A mixture model approach," Genome Biology, 3(5), research0022.1-0022.10.

Parmigiani, G., Garrett, E. S., Anbazhagan, R., and Gabrielson, E. (2002), "A statistical framework for expression-based molecular classification in cancer," Journal of the Royal Statistical Society B, 64, 717-736.

Petrone, S. (1999), "Bayesian density estimation using Bernstein polynomials," Canadian Journal of Statistics, 27, 105-126.

Richardson, S. and Green, P. J. (1997), "On Bayesian Analysis of Mixtures with an Unknown Number of Components," Journal of the Royal Statistical Society B, 59, 731-792.

Robert, C. and Rousseau, J. (2002), "A mixture approach to Bayesian goodness of fit," Tech. rep., CEREMADE.

Stollberg, J. Urschitz, J., Urban, Z., and Boyd, C. (2000), "A quantitative evaluation of Sage," Genome Research, 10, 1241-1248. 
Tadesse, M., Sha, N., Kim, S., and Vannucci, M. (2006), "Bayesian Inference for Gene Expression and Proteomics," in Do et al. (2006), chap. Identification of Biomarkers in Classification and Clustering of High-Throughput Data, pp. $1-91$.

Tadesse, M., Sha, N., and Vannucci, M. (2005), "Bayesian variable selection in clustering high-dimensional data," Journal of the American Statistical Association, 100, 602-617.

Zhang, L., Zhou, W., Velculescu, V., Kern, S., Hruban, R., Hamilton, S., Vogelstein, B., and Kinzler, K. (1997), "Gene Expression Profiles in Normal and Cancer Cells," Science, 276, 1268-1272. 